cortex revealed a significant peak in the number of $\mathrm{T}$ lymphocytes and neutrophils at $24 \mathrm{~h}$ compared with other time points $(P<0.05)$. Concentrating on this peak time for infiltration, a separate group of rats was pre-treated with S-PBN $24 \mathrm{~h}$ after TBI. S-PBN reduced the infiltration of ex vivo propagated immune cells by $50 \%$ compared with no treatment $(P<0.01)$. T-cell receptor $\alpha \beta$-positive $\mathrm{T}$ lymphocytes were decreased by $95 \%(P<0.01)$, and neutrophil infiltration was also attenuated, although not as dramatically.

The role of T-lymphocyte and neutrophil infiltration in neuroprotection following TBI is incompletely understood, but the results of this study show that inhibition of reactive oxygen species affects the level of infiltration. The authors conclude that inhibition of T-cell trafficking to the injured brain at the microvascular level could present a novel treatment approach to limiting secondary injury following TBI.

Original article Clausen F et al. (2007) T lymphocyte trafficking: a novel target for neuroprotection in traumatic brain injury. J Neurotrauma 24: 1295-1307

\section{Cerebellar hemorrhagic injury affects neurodevelopment in preterm infants}

Cerebellar hemorrhagic injury $(\mathrm{CHI})$ is increasingly recognized as a complication of premature birth; however, few studies have examined its potential role in the high prevalence of cognitive, learning and behavioral disabilities observed in premature infants. To evaluate the possible effect of $\mathrm{CHI}$ on neurodevelopment in ex-preterm infants, Limperopoulos et al. conducted a retrospective case-control study.

The researchers identified 51 premature infants with $\mathrm{CHI}$ (16 with additional supratentorial parenchymal injury) from their neonatal cranial ultrasonography records, and matched each isolated $\mathrm{CHI}$ case with a control infant from previous prospective studies.

Neurologic abnormalities were detected in $66 \%$ of infants with $\mathrm{CHI}$ compared with only $5 \%$ of control infants. In comparison with control infants, those with isolated $\mathrm{CHI}$ had lower mean scores for gross and fine motor functioning, expressive and receptive language and visual reception (Mullen Scales of Early Learning; $P<0.001$ for all), and lower scores for communication, daily living, socialization and motor functioning (Vineland Adaptive Behavior Scale; $P<0.001$ for all). The prevalence of neurodevelopmental abnormalities was no higher in infants with $\mathrm{CHI}$ and supratentorial parenchymal injury than in those with isolated $\mathrm{CHI}$, although neuromotor impairment was more severe. In addition, infants with $\mathrm{CHI}$ had a greater prevalence of positive screening tests for autism as well as for internalizing behavioral problems. The authors conclude that $\mathrm{CHI}$ in premature infants is associated with an increased risk of neurodevelopmental abnormalities, a fact that is currently under-recognized.

Original article Limperopoulos C et al. (2007) Does cerebellar injury in premature infants contribute to the high prevalence of long-term cognitive, learning, and behavioural disability in survivors. Pediatrics 120: 584-593

\section{APOE-associated accumulation of dementia in sporadic CJD}

A recent study has suggested that a positive family history of dementia (PFHD) is more common in patients with sporadic Creutzfeldt-Jakob disease (SCJD) than in controls, but the analysis included some patients with familial CJD, which might have skewed the results. To clarify the findings, Krasnianski et al. studied the incidence of PFHD in the parents, grandparents and siblings of 685 German patients with sCJD and 659 healthy controls matched for age and sex, and examined possible associated risk factors.

Twice as many SCJD patients as controls were found to have PFHD. After discounting the possibility that the difference could be attributable to relatives' ages, the authors looked for a possible genetic explanation. They investigated whether sCJD with PFHD was associated with the presence of the M129V polymorphism of the prion protein gene (PRNP), or with one of the six apolipoprotein E (APOE) alleles. The PRNP M129V polymorphism has been linked with dementia and $A P O E$ polymorphism is associated with Alzheimer's disease and possibly also vascular dementia, but the role of these genetic variants in CJD is unclear.

No significant difference in M129V polymorphism was found between SCJD with and SCJD without PFHD; however, $A P O E^{\star} 4$ allele frequency and the proportion of $A P O E^{\star} 4$ allele carriers were significantly higher, and the $A P O E^{\star} 3$ allele significantly rarer, in SCJD with PFHD than in controls or in SCJD without PFHD. 